See discussions, stats, and author profiles for this publication at: https://www.researchgate.net/publication/322409794

\title{
Formulations and Branch-and-Cut Algorithms for Production Routing Problems with Time Windows
}

Article in Transportmetrica A: Transport Science · January 2018

DOI: 10.1080/23249935.2018.1427157

CITATIONS

5

5 authors, including:

Yuzhuo Qiu

Nanjing University of Finance and Economics

19 PUBLICATIONS 233 CITATIONS

SEE PROFILE

Boris Goldengorin

University of Baltimore

146 PUBLICATIONS 1,109 CITATIONS

SEE PROFILE
142

P. M. Pardalos

University of Florida

373 PUBLICATIONS 8,703 CITATIONS

SEE PROFILE

Some of the authors of this publication are also working on these related projects:

Submodular optimization, binary search and truncation theorem will be able to change the models and algorithms in machine learning. View project

Tolerance Based Algorithms in Combinatorial Optimization View project 


\section{Formulations and branch-and-cut algorithms for production routing problems with time windows}

\section{Yuzhuo Qiu, Liang Wang, Xuanjing Fang, Panos M. Pardalos \& Boris Goldengorin}

To cite this article: Yuzhuo Qiu, Liang Wang, Xuanjing Fang, Panos M. Pardalos \& Boris Goldengorin (2018) Formulations and branch-and-cut algorithms for production routing problems with time windows, Transportmetrica A: Transport Science, 14:8, 669-690, DOI: 10.1080/23249935.2018.1427157

To link to this article: https://doi.org/10.1080/23249935.2018.1427157

Accepted author version posted online: 11 Jan 2018.

Published online: 26 Jan 2018.

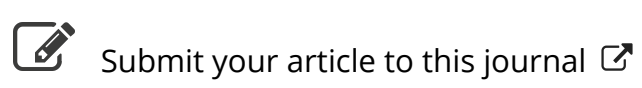

Џ Article views: 77

View Crossmark data ¿ 


\title{
Formulations and branch-and-cut algorithms for production routing problems with time windows
}

\author{
Yuzhuo Qiu (D) a,b , Liang Wang ${ }^{\mathrm{a}}$, Xuanjing Fang ${ }^{\mathrm{a}}$, Panos M. Pardalos ${ }^{\mathrm{b}}$ and \\ Boris Goldengorinc \\ a Jiangsu Key Laboratory of Modern Logistics, School of Marketing and Logistics Management, Nanjing \\ University of Finance and Economics, Nanjing, People's Republic of China; ${ }^{b}$ Department of Industrial and \\ Systems Engineering, Faculty of Engineering, University of Florida, Gainesville, FL, USA; ${ }^{C}$ Department of \\ Industrial and Systems Engineering, The Russ College of Engineering and Technology, Ohio University, \\ Athens, $\mathrm{OH}$, USA
}

\begin{abstract}
This paper presents a mixed integer optimization framework for incorporating time windows into production routing problems. This problem is a generalization of vehicle routing, inventory routing, and lot-sizing problems, and formulated as a mixed interlinear programming problem. An exact method within a branch-and-cut framework is developed to solve the model. Several families of valid cuts are adapted and a hybrid heuristic to obtain a good upper bound is also developed. The newly proposed $(I, S)$ inequalities link production variables with inventory variables. From the computational results, the effectiveness of the valid inequalities is proved. The newly proposed $(I, S)$ inequalities outperform previously related inequalities. The numerical results for the case study also show that the total cost results in an $11.6 \%$ decrease over a heuristic solution after applying the proposed model and algorithm.
\end{abstract}

\section{ARTICLE HISTORY}

Received 24 March 2017

Accepted 9 January 2018

\section{KEYWORDS}

Vehicle routing; inventory/production; deterministic; integer programming; algorithms; cutting plane

\section{Introduction}

Many real-world applications have widely shown that using computerized solution methods for the solution of vehicle routing problem (VRP) yields substantial savings in the global transportation costs (Toth and Daniele 2014; Chow and Nurumbetova 2015; You, Chow, and Ritchie 2016). Production routing problem (PRP) is a variant of VRP. This problem aims at optimizing decisions of production, inventory, distribution, and routing simultaneously (Adulyasak, Cordeau, and Jans 2015b). Solving such integrated optimization problems can contribute to finding better solutions in supply chain management, specifically in vendor managed inventory (VMI) and the practice of just-in-time (JIT) production and logistics.

In the past decade, the PRP was extended in various ways, such as multiple plants and a heterogeneous fleet of vehicles (Lei et al. 2006), uncapacitated production (Archetti et al. 2011), multiple homogeneous capacitated vehicles (Adulyasak, Cordeau, and Jans 2014b), and under demand uncertainty (Adulyasak, Cordeau, and Jans 2015a).

CONTACT Yuzhuo Qiu jadeqyz@gmail.com @ Jiangsu Key Laboratory of Modern Logistics, School of Marketing and Logistics Management, Nanjing University of Finance and Economics, Nanjing 210023, People's Republic of China 
Incorporation of delivery or supply time windows within the PRP is one of the future research directions (Adulyasak, Cordeau, and Jans 2015b; Díaz-Madroñero, Peidro, and Mula 2015). To the best of our knowledge, little effort has been made in this direction. Our aim is to bridge this gap by incorporating the still-missing time windows in production routing problems.

There has been extensive research in the PRP with heuristics: approximation algorithms (Chandra and Fisher 1994), decoupled heuristics (Fumero and Vercellis 1999), greedy randomized adaptive search procedures (Boudia, Louly, and Prins 2007), memetic algorithms (Boudia and Prins 2009), tabu search (Bard and Nananukul 2009b; Armentano, Shiguemoto, and Lokketangen 2011), adaptive large neighborhood heuristics Adulyasak, Cordeau, and Jans (2014a), iterative mixed integer programming (Absi et al. 2015), and particle swarm optimization (Kumar et al. 2016). Research on the exact algorithms has only been addressed in a few publications. For example, branch-and-price (Bard and Nananukul 2009a, 2010; Qiu, Qiao, and Pardalos 2017) and branch-and-cut algorithms for single product PRPs were investigated recently (Archetti et al. 2011; Adulyasak, Cordeau, and Jans 2014b).

The PRP involves combinatorial optimization of both delivery and routing decisions. Current branch-and-cut algorithms for multi-vehicle PRPs can only solve the instances up to 50 customers, 3 periods, and 3 vehicles in 2 hours by using parallel computing (Adulyasak, Cordeau, and Jans 2014b). However, supply chain participants often face with problems with longer planning periods or more vehicles. An exact algorithm to solve larger instances is still necessary, especially when practical concerns such as time windows are further incorporated into the original PRP.

A limitation in previous exact algorithms for the PRP is the lack of lot sizing problems (LSP)-related valid inequalities. The LSP is a major component of the PRP (Adulyasak, Cordeau, and Jans 2015b). LSP-related valid inequalities are thus expected to enhance the performance of exact methods to solve the PRP. In this paper, we aim to strengthen the formulation of the PRP with time windows (PRPTW) by these valid inequalities.

The contributions of the paper are summarized as follows. First, we introduce a realworld variant of the PRP which addresses customer delivery windows. Second, we formulate the PRPTW as a mixed integer linear programming (MILP) problem. Third, we introduce four families of valid inequalities to tighten the MILP problem and design a branch-and-cut algorithm to find an optimal solution. Moreover, the proposed $(I, S)$ inequalities outperform previous related valid cuts by linking inventory variables with production variables. Our computational results with the branch-and-cut algorithm show that the proposed valid inequalities can substantially improve the quality of lower bounds. The computational result from case study shows a more than $10 \%$ decrease in total costs. Our PRPTW model, algorithm, and computational results can serve as a stepping stone for further research of PRPs with startup times, change-over times, and other extensions (Adulyasak, Cordeau, and Jans 2015b).

The remainder of this paper is organized as follows. In Section 2, we provide a formal description of the problem. Several families of valid inequalities are then introduced in Section 3. Based on these valid inequalities and MILP formulation, we devise a branch-andcut algorithm in Section 4. We present computational results of our algorithm in Sections 5 and 6. Conclusions and discussions are presented in Section 7. 


\section{Problem description and model formulations}

We now introduce the notations used in our models. The MILP formulation with the Miller-Tucker-Zemlin (MTZ)-like constraints is then proposed, which is solvable for smalland medium-scale instances. For large-scale instances, another strengthened formulation with generalized capacity constraints and path inequalities is presented and used in the proposed branch-and-cut algorithm.

\subsection{Notations and problem description}

We define the PRPTW on a complete directed graph $G=\left(N_{0}, A\right)$ with sets of nodes $N_{0}=$ $N \cup\{0\}$ and $\operatorname{arcs} A=\left\{(i, j): i, j \in N_{0}, i \neq j\right\}$, respectively. The node set $N_{0}=N \cup\{0\}$ contains a set $N=\{1,2, \ldots, n\}$ of customers (retailers) and the plant (depot) represented by node 0 . Under limited production capacity $C$, a commodity is produced at the plant with a fixed cost of $f$ and a unit cost of $u$, and shipped to the retailers by a fleet of identical vehicles over a finite set $T$ of time periods.

In every period $t$, the service for each customer $i$ should start within a given time window $\left[a_{i t}, b_{i t}\right]$. The vehicle must arrive at customer $i$ no later than $b_{i t}$. If the vehicle arrives earlier than $a_{i t}$, the vehicle must wait until the earliest starting time for service. We assume that the vehicle traverses each arc with an average speed of $\mathcal{V}$, hence it takes time $c_{i j} / \mathcal{V}$ to transport over an arc $(i, j)$ of length $c_{i j}$. Assume it takes time $\gamma_{i}$ to serve a customer $i$, it thus takes at least time $\tau_{i j}=c_{i j} / \mathcal{V}+\gamma_{i}$ to serve customer $i$ and reach the next customer $j$. When we formulate the MILP model, we assume the transport cost over an arc $(i, j)$ of length $c_{i j}$ is also $c_{i j}$ to keep the formulation simple. Incorporation of a unit transport cost per unit length is straightforward and will not change the formulation much. The average speed of $\mathcal{V}$ is also left out in the following formulation for the same simplicity reasons.

The inventory capacities $L_{i}$ are imposed at the end of a period, after a delivery is made from the plant to a customer and the customer's demand is satisfied. Given the initial inventory levels and the unit inventory holding costs $h_{i}$ of the product at node $i$, the PRPTW is to determine the production, shipment, and inventory quantities as well as the set of routes in each period to minimize the total production, inventory, and routing costs and meet the customers' demand within time windows requirements.

Since the Vehicle Routing Problem with Time Windows (VRPTW) (Savelsbergh 1985) is $\mathcal{N} P$-hard, and the PRPTW reduces to multi-period VRP when production variables are constants, and delivery quantities are known beforehand, one may conclude that the PRPTW is also $\mathcal{N} P$-hard. Note that finding a feasible solution to the VRPTW for a fixed number of vehicles is an $\mathcal{N} P$-complete problem. Thus finding a feasible solution to the PRPTW is also an $\mathcal{N} P$-complete problem.

Notations for production, inventory, and routing parameters and decision variables are as follows.

\section{Notations and Parameters}

fixed production setup cost

$u \quad$ unit production cost

$h_{i} \quad$ unit inventory holding cost at node $i$

$c_{i j} \quad$ transportation cost from node $i$ to node $j$ 
$\gamma_{i}$

$d_{i t}$

$a_{i t}$

$b_{i t}$

$M_{i j t}$

$B_{t}$

$E_{i t}$

$|T|$

$Q$

K

$L_{i}$

C

$\delta_{t}^{+}(S)$

$\delta_{t}^{-}(S)$

$\mathcal{Z}^{+} \quad$ set of positive integers

$P \quad$ set of paths

$P_{G} \quad$ set of all minimal infeasible paths in $\mathrm{G}$

$p$

$N(p)$

$|N(p)|=I$

$A(p)$

$|A(p)|=I-1$

service time at node $i$

demand of customer (retailer) $i$ in period $t$ i

$\max \left\{b_{i t}+\gamma_{i}+\tau_{i j}-a_{j t}, 0\right\}$

$=\min \left\{C, \sum_{k=t}^{|T|} \sum_{i \in N} d_{i k}\right\}$

$=\min \left\{L_{i}, \sum_{k=t}^{|\bar{T}|} d_{i k}\right\}$

number of time periods

vehicle capacity

number of available vehicles

maximum or target inventory level at node $i$

production capacity $S, j \notin S\}$, and $\delta_{t}^{+}(i):=\delta_{t}^{+}(\{i\})$ $S\}$, and $\delta_{t}^{-}(i):=\delta_{t}^{-}(\{i\})$

path $p=\left(v_{1}, v_{2}, \ldots, v_{l}\right), p \in P$

node set of path $p=\left(v_{1}, v_{2}, \ldots, v_{l}\right)$

number of nodes on path $p=\left(v_{1}, v_{2}, \ldots, v_{l}\right)$

arc set of path $p=\left(v_{1}, v_{2}, \ldots, v_{l}\right)$

opening time, i.e. the earliest possible starting time for servicing customer

closing time, i.e. the latest possible time for servicing customer $i$

set of arcs leaving from the node set $S$ in period $t, \delta_{t}^{+}(S)=\{(i, j) \in A: i \in$

set of arcs entering the node set $S$ in period $t, \delta_{t}^{-}(S)=\{(i, j) \in A: i \notin S, j \in$

Decision Variables

$q_{t} \quad$ production quantity in period $t$

$l_{\text {it }} \quad$ inventory at node $i$ at the end of period $t$

$r_{i t} \quad$ shipment quantity to customer (retailer) $i$ in period $t$

$x_{i j t} \quad$ binary variable if arc $(i, j)$ is traversed in period $t, 0$ otherwise

$y_{t} \quad$ binary variable, equal to 1 if the product is set up for production in period $t, 0$ otherwise

$z_{i t} \quad$ integer variable if vertex $i$ is visited in period $t, 0$ otherwise

$v_{i t} \quad$ total quantity carried on the vehicle at retailer $i$ in period $t$ on a feasible route

$W_{\text {it }} \quad$ start of service time at retailer $i$ in period $t$ on a feasible route.

\subsection{A MILP formulation for the PRPTW: formulation I}

Let $x_{t}(S)=\sum_{i \in S, j \in S} x_{i j t}, r_{t}(S)=\sum_{i \in S} r_{i t}$ and $z_{t}(S)=\sum_{i \in S} z_{i t}$, using the notations given in Section 2.1, we formulate the PRPTW as follows:

$$
\min \sum_{t \in T}\left(u q_{t}+f y_{t}+\sum_{i \in N_{0}} h_{i} l_{i t}\right)+\sum_{t \in T} \sum_{(i, j) \in A} c_{i j} x_{i j t}
$$


subject to

$$
\begin{aligned}
& I_{0, t-1}+q_{t}=I_{0 t}+\sum_{i \in N} r_{i t}, \quad \forall t \in T, \\
& l_{i, t-1}+r_{i t}=l_{i t}+d_{i t}, \quad \forall i \in N, t \in T, \\
& q_{t} \leq B_{t} y_{t}, \quad \forall t \in T \\
& l_{i t} \leq L_{i}, \quad \forall i \in N_{0}, t \in T \text {, } \\
& r_{i t} \leq E_{i t} z_{i t}, \quad \forall i \in N, t \in T, \\
& \sum_{j \in N_{0}} x_{i j t}=z_{i t}, \quad \forall i \in N_{0}, t \in T \\
& \sum_{j \in N_{0}} x_{i j t}=\sum_{j \in N_{0}} x_{j i t}, \quad \forall i \in N_{0}, t \in T, \\
& v_{i t}-v_{j t}+Q x_{i j t} \leq Q-r_{j t}, \quad \forall i, j \in N, t \in T, \\
& W_{i t}-W_{j t}+M_{i j t} x_{i j t} \leq M_{i j t}-\tau_{i j,} \quad \forall i, j \in N, t \in T, \\
& q_{t} \geq 0, \quad y_{t} \in\{0,1\}, \quad \forall t \in T, \\
& l_{i t} \geq 0, \quad r_{i t} \geq 0, \quad \forall i \in N_{0}, t \in T, \\
& z_{i t} \in\{0,1\}, \quad \forall i \in N, t \in T, \\
& z_{0 t} \in \mathcal{Z}^{+}, \quad z_{0 t} \leq K, \quad \forall t \in T, \\
& x_{i j t} \in\{0,1\}, \quad \forall i, j \in N_{0}, t \in T . \\
& r_{i t} \leq v_{i t} \leq Q, \quad \forall i \in N, t \in T, \\
& 0 \leq W_{i t} \leq b_{i t}, \quad \forall i \in N, t \in T
\end{aligned}
$$

The objective function (1) measures the operational cost of production, inventory and routing. Constraints (2) and (3) enforce inventory flow balance at the plant and retailer for two sequential time periods $t-1$ and $t$, respectively. Constraints (4) ensure that the binary 
variables of setup $y_{t}=1$ if production takes place. These constraints limit the production quantity to the minimum of production capacity and the total demand in the remaining time periods. Constraints (5) impose inventory limits both at the plant and the retailers. Constraints (6) permit a positive delivery quantity to node $i$ in period $t$ if this node is visited in period $t$. Constraints (7) ensure $z_{i t}>0$ if a truck leaves from retailer or depot $i$. Constraints (8) enforce vehicle flow conservation. Constraints (9) are MTZ constraints of vehicle capacity for the PRPTW. The MTZ formulation for the TSP reduces the number of constraints at the cost of weaker lower bounds than subtour elimination constraints (Miller, Tucker, and Zemlin 1960). Constraints (10) are MTZ-like constraints of time windows for the PRPTW. Note that in 1-hour CPU time, the largest PRPTW instance based on the Formulation I and solved by the proposed branch-and-cut algorithm is 25 customers and 3 time periods. Hence, it is necessary to strengthen the formulation and develop a corresponding branch-and-cut algorithm.

\subsection{A strengthened MILP formulation for the PRPTW: formulation II}

As indicated in Section 2.2, the lower bound obtained by MTZ formulation for the PRPTW is rather weak. To further strengthen the formulation, we replace the MTZ-like constraints (9) and (10) with the following constraints.

$$
\begin{gathered}
Q x_{t}(S) \leq Q z_{t}(S)-r_{t}(S), \quad \forall S \subseteq N,|S| \geq 2, t \in T, \\
\sum_{(i, j) \in A(p)} x_{i j t} \leq|A(p)|-1, \quad \forall p \in P_{G}, t \in T .
\end{gathered}
$$

Constraints (18) serve as the subtour eliminations and vehicle capacity constraints. Constraints (19) are known as the incompatible path inequalities or path inequalities to forbid the generation of paths that violate time windows and vehicle capacity requirements. In the following branch-and-cut algorithm, these constraints are first relaxed from the linear programming (LP) relaxation and then added back as cutting planes.

The strengthened PRPTW formulation (1)-(8), (11)-(15), (18) and (19) (Formulation II) now serves as the basis for solving large size instances with the proposed branch-and-cut algorithm.

\section{Valid inequalities}

In this section, we introduce several families of valid inequalities to strengthen the LP relaxation of the PRPTW formulation (1)-(15). The proof of these valid inequalities is left in the online supplement. For the sake of brevity, we use similar notations to describe the inequalities related to the VRPTW by dropping the subscript $t$ of the PRPTW notations and describe the inequalities related to the LSP by dropping the subscript $i$ of the PRPTW notations.

\subsection{Strengthened reachability inequalities}

The Reachability Inequalities (RIs) are rather efficient for the VRPTW (Lysgaard 2006). For any node $i \in N$, let $R_{i}^{-}$and $R_{i}^{+}$denote the reaching and reachable set of arcs for node $i$, respectively. 
Definition 3.1 (Lysgaard 2006): For any customer $i \in N$, the reaching arc set $R_{i}^{-} \subset A$ is defined as the minimum arc set such that any feasible path $(0, \ldots, i)$ lies inside $R_{i}^{-}$, i.e. $(j, k) \in$ $A$ for each $\operatorname{arc}(j, k)$ on the feasible path.

Definition 3.2 (Lysgaard 2006): For any customer $i \in N$, the reachable arc set $R_{i}^{+} \subset A$ is defined as the minimum arc set such that any feasible path $(i, \ldots, 0)$ lies inside $R_{i}^{+}$, i.e. $(j, k) \in$ $A$ for each $\operatorname{arc}(j, k)$ on the feasible path.

For any $S \subseteq N$ and $i \in S$, the following Rls

$$
x\left(\delta^{-}(S) \cap R_{i}^{-}\right) \geq 1
$$

and

$$
x\left(\delta^{+}(S) \cap R_{i}^{+}\right) \geq 1
$$

are valid for the VRPTW (Lysgaard 2006). We extend the above definitions and valid inequalities to a multi-period version as follows.

Definition 3.3: For any customer $i \in N$, the reaching arc set $R_{i t}^{-} \subset A_{t}$ is defined as the minimum arc set such that any feasible path $(0, \ldots, i)$ lies inside $R_{i t}^{-}$, i.e. $(j, k) \in A_{t}$ for each arc $(j, k)$ on the feasible path.

Definition 3.4 (Lysgaard 2006): For any customer $i \in N$, the reachable arc set $R_{i}^{+} \subset A_{t}$ is defined as the minimum arc set such that any feasible path $(i, \ldots, 0)$ lies inside $R_{i}^{+}$, i.e. $(j, k) \in A_{t}$ for each arc $(j, k)$ on the feasible path.

Moreover, we tighten the generalized RIs for the PRPTW as follows.

Proposition 3.5: Let $R_{i t}^{-}$and $R_{i t}^{+}$be the reaching and reachable set of arcs to node $i$ at period $t$, respectively. The following strengthened reachability inequalities

$$
Q x_{t}\left(\delta_{t}^{-}(i) \cap R_{i t}^{-}\right) \geq r_{i t}, \quad \forall i \in S, S \subseteq N, t \in T
$$

and

$$
Q x_{t}\left(\delta_{t}^{+}(i) \cap R_{i t}^{+}\right) \geq r_{i t}, \quad \forall i \in S, S \subseteq N, t \in T
$$

are valid for the PRPTW.

\subsection{Strengthened $(I, S)$ inequalities}

LSPs are also essential to the PRPTW. When there is only one supplier but no retailers, and when costs are Wagner-Whitin costs, i.e. $u_{t}+h_{t}>u_{t+1}$, the following $(I, S)$ inequalities are valid for the LSP (Pochet and Wolsey 2006)

$$
I_{t-1} \geq \sum_{j=t}^{\eta} d_{j}\left(1-y_{t}-y_{t+1}-\cdots-y_{j}\right), \quad \forall \eta: t<\eta \leq|T|, t \in T
$$

where $d_{j}$ is the demand for period $j$ at the supplier, $y_{j}$ is the binary set-up variable for period $j$. Note that in our model, $u_{t}=u_{t+1}=u$. Therefore, our model features with Wagner-Whitin 
costs. In the PRPTW, we must also deal with the demand and inventories at the retailers. Thus, inspired by the aggregated subtour elimination constraints (Adulyasak, Cordeau, and Jans 2014a), we have the following proposition.

Proposition 3.6: The following strengthened $(I, S)$ inequalities

$$
\sum_{i \in N_{0}} I_{i, t-1}+\sum_{j=t+1}^{\eta} \sum_{k=j}^{\eta} \sum_{i \in N} d_{i k} y_{j} \geq \sum_{j=t}^{\eta} \sum_{i \in N} d_{i j}\left(1-y_{t}\right), \quad \forall \eta: t<\eta \leq|T|, t \in T
$$

are valid for the PRPTW.

Note that the proposed $(I, S)$ inequalities are different from the following inventoryrelated valid inequalities (Archetti et al. 2011)

$$
\left(\sum_{j=0}^{s} \delta_{i, t-j}\right)\left(1-\sum_{k=0}^{s} z_{i, t-k}\right) \leq l_{i, t-s-1}^{\mathrm{d}}, \quad \forall i \in N, t \in T, 0 \leq s \leq t-1,
$$

where constraints (26) guarantee that in each time instant, product inventory is enough to cover accumulated delivery demand. The difference lies in that the proposed $(I, S)$ inequalities (25) link inventory and production variables, whereas constraints (26) only deal with inventory variables.

\subsection{Logical inequalities}

The formulations (1)-(8), (11)-(15), (18) and (19) can be strengthened by adding the following valid inequalities, known as logical inequalities (LIs):

$$
\begin{gathered}
z_{i t} \leq z_{0 t}, \quad \forall i \in N, t \in T, \\
z_{i t} \leq r_{i t}, \quad \forall i \in N, t \in T, \\
l_{i|T|}=0, \quad \forall i \in N_{0} .
\end{gathered}
$$

The inequalities (27a) and (27c) have appeared in the PRP literature (Archetti et al. 2007; Adulyasak, Cordeau, and Jans 2014a).

The inequalities (27b) we propose for the PRPTW ensure that the number of visits to node $i$ equals zero if no delivery occurs at node $i$. This is not guaranteed by the original formulation but implied by an optimal solution if the triangular inequality $c_{i k} \leq c_{i j}+c_{j k}, \forall i, j, k \in N_{0}$ holds strictly. When the triangular inequality does not hold strictly, we can not assert (27b) hold because visiting customer $j$ without demand does not incur extra transportation cost, if $c_{i k}=c_{i j}+c_{j k}$. Visiting customer $j$ does not form a detour in this case. Our computational study shows that adding (27b) makes the lower bounds tighter in the instances for which triangular inequality holds strictly.

In addition, valid inequalities preventing stockout are usually added prior to solving the problem. Denote by $t^{\prime}$ the earliest time period that a production must be made, we know that $t^{\prime}=\arg \min _{t \in T}\left\{\sum_{i \in N} \max \left\{0, \sum_{j=1}^{t} d_{i j}-l_{i 0}\right\}-I_{00}>0\right\}$. Then, denote by $t^{\prime \prime}$ the earliest 
time period such that at least one customer must be replenished to avoid stockout, we know that $t^{\prime \prime}=\arg \min _{i \in N} t_{i}^{\prime}$, with $t_{i}^{\prime}=\arg \min _{t \in T}\left\{\sum_{j=1}^{t} d_{i j}-l_{i 0}>0\right\}$. Finally, denote by $\kappa=\sum_{i \in N} \max \left\{0, \sum_{j=1}^{t^{\prime \prime}} d_{i j}-l_{i 0}\right\}$ the minimum shipping quantity to prevent stockout. We have the following valid inequalities (Archetti et al. 2007, 2011; Adulyasak, Cordeau, and Jans 2014b)

$$
\begin{gathered}
\sum_{t=1}^{t^{\prime}} y_{t} \geq 1 \\
\sum_{t=1}^{t^{\prime \prime}} z_{0 t} \geq\left\lceil\frac{\kappa}{Q}\right\rceil,
\end{gathered}
$$

for the PRPTW.

We also propose the following logical valid inequalities

$$
\begin{gathered}
\sum_{t=1}^{t_{i}^{\prime}} z_{i t} \geq 1, \quad \forall i \in N \\
\sum_{t=1}^{t_{i}^{\prime}} r_{i t} \geq \sum_{j=1}^{t_{i}^{\prime}} d_{i j}-l_{i 0}, \quad \forall i \in N
\end{gathered}
$$

The above constraints are largely ignored in the PRP literature. Our computational study indicates that these inequalities can tighten the lower bounds.

\subsection{VRPTW-related inequalities}

We propose the following generalized Asymmetric-Traveling-Salesman-Problem-withTime-Windows (ATSPTW) inequalities for the PRPTW

$$
\begin{aligned}
& Q\left(x_{v_{k} v_{1} t}+\sum_{h=2}^{k} x_{v_{h-1} v_{h} t}+2 \sum_{h=2}^{k-1} x_{v_{h} v_{1} t}+\sum_{h=3}^{k-1} \sum_{j=2}^{h-1} x_{v_{h} v_{j} t}\right) \\
& \leq Q z_{t}(N(p))-r_{t}(N(p)), \quad \forall t \in T
\end{aligned}
$$

and

$$
\begin{aligned}
& Q\left(x_{v_{1} v_{k} t}+\sum_{h=2}^{k} x_{v_{h} v_{h-1} t}+2 \sum_{h=2}^{k-1} x_{v_{1} v_{h} t}+\sum_{h=3}^{k-1} \sum_{j=2}^{h-1} x_{v_{j} v_{h} t}\right) \\
& \leq Q z_{t}(N(p))-r_{t}((N(p)), \quad \forall t \in T
\end{aligned}
$$

where $z_{t}(N(p))=\sum_{v_{i} \in p} z_{v_{i} t}, r_{t}(N(p))=\sum_{v_{i} \in p} r_{v_{i} t}$ and $k \geq 3$.

We also introduce generalized tournament inequalities (GTIs) for the PRPTW as follows:

$$
\sum_{i=1}^{k-1} \sum_{j=i+1}^{k} x_{v_{i} v_{j} t} \leq k-2(=|A(p)|-1), \quad \forall t \in T
$$




\section{Branch-and-cut algorithm}

Since the VRP and the LSP within the PRPTW usually comprise of exponential numbers of subtour elimination constraints, it is natural to resort to dynamic column or row generation. In this section by using the MILP formulation and valid inequalities described in previous sections, we devise a branch-and-cut algorithm to solve the PRPTW exactly. A description of our branch-and-cut algorithm is as follows.

\subsection{Linear programming relaxation and a priori cutting planes}

The linear programming relaxation of the MILP formulation without the generalized capacity and path constraints is solved at each node of the branch-and-cut tree. Except for experiments to test the effectiveness of the valid inequalities, logical valid inequalities are added a priori to the linear programming relaxation of the MILP formulation. Note that the number of LSP-related valid inequalities usually increases exponentially with time periods. Thus adding LSP-related inequalities a priori will increase computational complexity when the number of time periods is relatively large.

\subsection{Separation procedures}

We adapt the well-known connected component-based separation procedures (Naddef and Rinaldi 2001; Bard, Kontoravdis, and Yu 2002; Kallehauge, Boland, and Madsen 2007) for GSECs (18). The auxiliary graph for each period is defined by arcs with nonzero values. Besides capacity inequalities, we also adapt the separation heuristic (Pochet and Wolsey 2006) for generalized $(I, S)$ inequalities, heuristics (Fischetti and Toth 1997) for ATSPTW inequalities, and separation techniques (Bard, Kontoravdis, and Yu 2002; Lysgaard 2006) for other VRPTW inequalities.

In all the adaptation, customers with positive values of variable $z_{i t}$ are included in and those with zero values of variable $z_{i t}$ are excluded from the resulting networks in the corresponding period $t$.

\subsection{Separation strategy}

We treat the root node of the branching tree differently from the other nodes (Lysgaard, Letchford, and Eglese 2004). If the optimal LP solution at the root node is fractional, we call the above-mentioned separation procedures for generalized capacity inequalities (18), the generalized $(I, S)$ inequalities (25), ATSPTW inequalities, and other VRPTW inequalities to search for violated cuts by the LP optimal solution. If at least one such cut is found, we add the violated constraints and the LP is reoptimized. The optimization and separation cycle of valid constraints iterates until no more violated inequalities are found. At other nodes, rather than the root node, if the solution is fractional, we branch. If the solution at the root node and other nodes is integer, we use the lazy constraints call-back (defined in C++ API of IBM ILOG CPLEX) containing the generalized capacity inequalities (12), the generalized tournament inequalities(32), and strengthened reachability inequalities (22) and (23) to check for violations (capacity and/or time windows).

\subsection{Branching strategy}

Three sets of binary or integer variables can be prioritized to branch in the PRPTW. We test different orders of variables to branch on. Computational experiments show that priority 
should be given first to $z_{i t}$, then $y_{t}$, and finally $x_{i j t}$ variables. An effective strategy of branching on the number of vehicles $z_{0 t}=\sum_{j \in N_{0}} x_{0 j t}$ in the VRP literature (Naddef and Rinaldi 2001; Lysgaard, Letchford, and Eglese 2004) corresponds to branching on $z_{0 t}$ variables in the PRPTW, and means branching on cut-set. Hence, we always branch on $z_{0 t}$ first before we branch on other $z_{i t}, i>0$. Our computational study shows that this special branching order works much better than the branching order of $y_{t}, z_{i t}$, and $x_{i j t}$ variables (Adulyasak, Cordeau, and Jans 2014a).

Our branch-and-cut algorithm uses a mixed strategy of most infeasibility and bestbound-first as the variable-and-node-selection rule (Lysgaard, Letchford, and Eglese 2004). The variable closest to 0.5 and the node with the largest lower bound are always processed first. This strategy combines the heuristic consideration and the fact that best-boundfirst search leads to the smallest possible branch-and-cut tree (Naddef and Rinaldi 2001; Lysgaard, Letchford, and Eglese 2004). Note that default strong branching strategy (Achterberg, Koch, and Martin 2005) in CPLEX is also switched on.

\subsection{Heuristics}

To speed up the solution process, we implement a hybrid heuristic method. The heuristic combines the ideas of large neighborhood search, local search, tabu search, and simulated annealing. We outline the heuristic in Algorithm 1.

The heuristic contains two phases, namely, construction and improvement. In the first phase, we construct initial solutions for the PRPTW by solving two decomposed problems of production-distribution and the VRPTW (Adulyasak, Cordeau, and Jans 2014b). The formulation is similar. However, the calculation of visiting cost is modified. In the previous study (Adulyasak, Cordeau, and Jans 2014b),

$$
\sigma_{i}=\min \left(2 c_{0 i,} \min _{j, k \in N_{0, j} \neq k}\left(c_{j i}+c_{i k}\right)\right) .
$$

We modify the approximate cost of visiting customer $i$ as

$$
\sigma_{i}=\min \left(2 c_{0 i,} \min _{j, k \in N, j \neq k} 0.5\left(c_{i j}+c_{i k}\right)\right) .
$$

The total visiting cost is probably closer to the actual routing cost with our modification. The reason is that, what we need in the formulation of the production-distribution subproblem is an estimate of the routing cost. We divide the estimated routing cost and attribute the cost to each customer to get the 'visiting cost'. Without the multiplication by 0.5 , we will approximately double the routing cost because each possible arc would be counted twice (once for each of its endpoints) in the final possible routes.

We use the Solomon insertion heuristic (Solomon 1987) to quickly obtain a solution of the VRPTW subproblem. We fix the shipment quantity variables $r_{i t}$ and site-visiting indicator variables $z_{i t}$ according to the solution of the solved production-distribution subproblem which could be formulated as follows:

$$
\min \sum_{t \in T}\left(u q_{t}+f y_{t}+\sum_{i \in N_{0}} h_{i} l_{i t}\right)+\sum_{t \in T} \sum_{i \in N} \sigma_{i} z_{i t}
$$




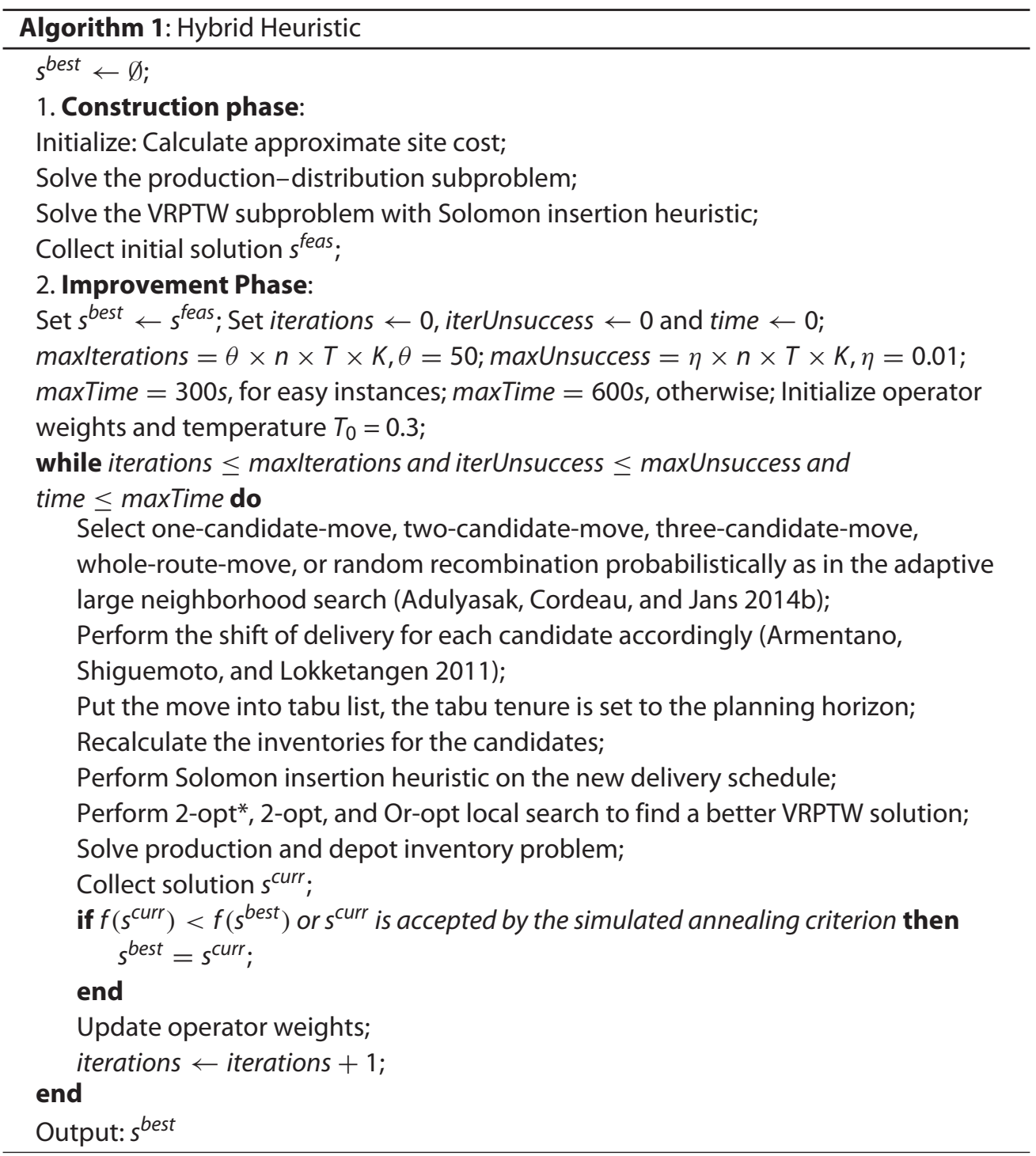

subject to (2)-(6), (11)-(14), (16) and (17). The initial solutions we get in this way are better than those using the original formulation (Adulyasak, Cordeau, and Jans 2014b; Armentano, Shiguemoto, and Lokketangen 2011).

In the second phase, we adopt a three-step procedure to improve the solutions. In the first step, we pick two, three, or whole-route customers in a period at a time and shift their deliveries to another period. We allow shifting to both the previous and next periods. The candidate customer-time pairs are selected adaptively (Adulyasak, Cordeau, and Jans 2014b). The transferring amounts of shipment are calculated accordingly (Armentano, Shiguemoto, and Lokketangen 2011). We apply a simulated annealing criterion to decide whether a local search solution should be accepted. The worse solution is accepted with probability $\exp \left((-100 / T)\left(f\left(s^{\text {curr }}\right)-f\left({ }^{\text {best }}\right)\right) / f\left({ }^{\text {best }}\right)\right)$, with $T=T_{k}=0.095 T_{k-1}$. The 
best move so far is recorded in the tabu list. The tabu tenure equals the planning horizon of the PRPTW. We incorporate tabu search because we want to avoid cycles when selecting candidate moves, and we add simulated annealing because we want to incorporate some randomness in the search process. The computational results show that the incorporation of tabu search and simulated annealing results in increase of the upper bound by $9.85 \%$ and $3.23 \%$ at most, respectively.

In the second step, we recalculate the inventories when all the shifts of shipment in the first step are carried out. We then solve the VRPTW for each period by taking the shipment quantities as demands for customers. The VRPTWs are solved first by the Solomon insertion heuristic (Solomon 1987). 2-opt, Or-opt, and 2-opt* local search heuristics (Toth and Daniele 2014) are then used to improve the VRPTW solutions.

In the third step, we solve the production and inventory problem at the depot by solving the corresponding mathematical programming model directly.

The above three-step search procedure terminates when it reaches maximum iterations or maximum time limits, or when the iteration without updating the incumbent solution reaches a limit. The resulting solution is provided as the initial solution to the branch-andcut algorithm.

\section{Computational results}

The experiments of this section were run using a laptop with Intel Core 2 Duo CPU P8600 of $2.40 \mathrm{GHz}$ under Ubuntu 14.04 (64 bits) with 4GB RAM. We implemented the branch-and-cut algorithm based on C++ API of IBM ILOG CPLEX version 12 release 6.

\subsection{Instance generation}

We adapt the Solomon benchmark (Solomon 1987) for the VRPTW to generate instances for the PRPTW. The coordinate, demand $d_{i}$ and service time of each customer, vehicle capacity $Q$, and number of vehicles available $K$ are taken from the Solomon benchmark. We assume that $d_{i t}=d_{i}$. We apply the time window of each customer directly for the first period. For later periods, the time windows are shifted to start from the end of previous period, i.e. the latest time a vehicle should return to the depot in the previous period. Other parameters for generating instances are listed in Table 1.

Note that Solomon benchmarks contain three classes of problems, namely, clustered (C), random (R), and randomly clustered (RC). Each has two subclasses. Random instances turn out to be the hardest to solve. We draw two instances from each subclass of different sizes in the following computational experiments. We test from 25 to 100 customers, 3 periods. For each problem size and instance, we generate 5 random cases. This process results in 300 instances totally. Details of these instances are provided in the online supplement.

\subsection{Data preprocessing}

After generating the instance, we tighten the time windows of each customer first. Then we calculate the reaching arc set $R_{i}^{-}$and the reachable arc set $R_{i}^{+}$for each customer. By definition (Lysgaard 2006), the reaching arc set $R_{i}^{-}$is the minimum arc set on any feasible path $(0, \ldots, i)$. The reachable arc set $R_{i}^{+}$is defined as the minimum arc set on any feasible 
Table 1. Generation of instances.

\begin{tabular}{lc}
\hline $\begin{array}{l}\text { Opening time at the plant and customers } \\
\text { in period } t>1\end{array}$ & $a_{i t}=a_{i, t-1}+b_{00}, \forall i \in N_{0}, \forall t>1$ \\
$\begin{array}{l}\text { Closing time at the plant and customers } \\
\text { in period } t>1\end{array}$ & $b_{i t}=b_{i, t-1}+b_{00}, \forall i \in N_{0}, \forall t>1$ \\
The transportation cost $c_{i j}$ from node $i$ to node $j$ & $c_{i j}=\operatorname{round}\left(\sqrt{\left(\chi_{i}-\chi_{j}\right)^{2}+\left(\psi_{i}-\psi_{j}\right)^{2}}\right)$ \\
with coordinates $\left(\chi_{i}, \psi_{i}\right)$ and $\left(\chi_{j}, \psi_{j}\right)$ & $C=\sum_{t} \sum_{i} d_{i}$ \\
Production capacity at the plant & $L_{0}=C / 2$ \\
Inventory capacity at the plant & $L_{i}=|T| * d_{i}$ \\
Inventory capacity at customers & $I_{i 0} \in[1,|T|-1] * d_{i}$ \\
Initial inventory at customers & $I_{00}=0$ \\
Initial inventory at the plant & $u \in[0.2,2]$ \\
Unit cost of production & $h_{i} \in[0.1,1]$ \\
Unit inventory holding cost at customers & $h_{0}=0.1$ \\
Unit inventory holding cost at the plant & $f=1000 h_{0}$ \\
Production set-up cost at the plant &
\end{tabular}

path $(i, \ldots, 0)$. Subsequently, we can identify an incompatible node pair $(i, j)$ by confirming the arcs between the node pairs are neither in $R_{j}^{-}$nor in $R_{i}^{-}$. We eliminate edge variables between incompatible node pairs.

We extend the time window reduction techniques (Bard, Kontoravdis, and Yu 2002; Kallehauge, Boland, and Madsen 2007) to a multi-period version which is described as follows.

\subsubsection{Opening time adjustment}

We use the following extended equations to adjust opening time of customer $k$ whose reaching or reachable sets (Lysgaard 2006) are not empty, respectively, at time $t$.

$$
\begin{gathered}
a_{k t}:=\max \left\{a_{k t}, \min _{(i, k) \in A}\left\{a_{i t}+c_{i k}\right\}\right\}, \quad \forall t \in T, \forall k \in N_{0}, \delta_{t}^{-}(k) \neq \varnothing \\
a_{k t}:=\max \left\{a_{k t}, \min \left\{b_{k t}, \min _{(k, j) \in A}\left\{a_{j t}-c_{k j}\right\}\right\}\right\}, \quad \forall t \in T, \forall k \in N_{0}, \delta_{t}^{+}(k) \neq \emptyset
\end{gathered}
$$

\subsubsection{Closing time adjustment}

Likewise, we use the following extended equations to adjust closing time of customer $k$ whose reaching or reachable sets are not empty, respectively, at time $t$.

$$
\begin{gathered}
b_{k t}:=\min \left\{b_{k t}, \max \left\{a_{k t}, \max _{(i, k) \in A}\left\{b_{i t}+c_{i k}\right\}\right\}, \quad \forall t \in T, \quad \forall k \in N_{0}, \delta_{t}^{-}(k) \neq \emptyset\right. \\
b_{k t}:=\min \left\{b_{k t}, \max _{(k, j) \in A}\left\{b_{j t}-c_{k j}\right\}\right\}, \quad \forall t \in T, \forall k \in N_{0}, \delta_{t}^{+}(k) \neq \emptyset
\end{gathered}
$$

\subsection{Results and discussion}

The experiments on the effects of the valid inequalities are conducted on the instances with $n=25 \rightarrow 100$ and $|T|=3$. The average results are shown in Tables 2 and 3 . 
Table 2. Effects of valid inequalities on average lower bounds at the root node.

\begin{tabular}{lcccccrr}
\hline Instance & With 1' (\%) & With 1 (\%) & With 2 (\%) & With 3 (\%) & With 4 (\%) & With 5 (\%) & All (\%) \\
\hline C.25-t3 & -0.02 & -0.04 & 2.84 & 0.18 & 0.07 & 1.72 & 8.18 \\
R.25-t3 & 0.00 & 0.00 & 6.14 & 0.52 & 0.00 & 3.70 & 14.97 \\
RC.25-t3 & 0.00 & 0.00 & 6.36 & 0.52 & 0.00 & 3.51 & 15.37 \\
C.30-t3 & 3.23 & 7.01 & 4.07 & 0.05 & 0.13 & 3.41 & 13.73 \\
R.30-t3 & 0.00 & 0.00 & 8.81 & 0.62 & 0.03 & 6.20 & 23.84 \\
RC.30-t3 & 0.00 & 0.00 & 11.20 & 0.60 & 0.02 & 6.36 & 23.95 \\
C.40-t3 & 1.72 & 4.71 & 1.98 & 0.22 & 0.02 & 2.58 & 8.26 \\
R.40-t3 & 2.19 & 4.78 & 8.21 & 0.68 & -0.18 & 3.55 & 15.41 \\
RC.40-t3 & 3.26 & 4.68 & 8.06 & 0.87 & -0.18 & 3.36 & 14.75 \\
C.50-t3 & 2.92 & 9.58 & 5.55 & 0.17 & -0.10 & 4.69 & 19.14 \\
R.50-t3 & 7.42 & 11.12 & 10.88 & 0.04 & 0.06 & 4.56 & 29.58 \\
RC.50-t3 & 5.52 & 10.91 & 17.36 & 0.06 & 0.20 & 4.40 & 29.96 \\
C.100-t3 & 4.32 & 7.10 & 2.91 & -0.03 & -0.03 & 4.00 & 14.45 \\
R.100-t3 & 5.59 & 9.76 & 8.68 & 0.08 & -0.08 & 4.49 & 23.16 \\
RC.100-t3 & 4.08 & 9.74 & 12.36 & 0.09 & -0.06 & 4.39 & 24.35 \\
\hline
\end{tabular}

Notes: The comparing base is the lower bound (LB) obtained from the LP relaxation of the MILP for the PRPTW, including only subtour elimination and incompatible path inequalities. Column With $7^{\prime}$ indicates adding inventory-related inequalities (Archetti et al. 2011) only upon the base LB. Column With 1 indicates adding generalized $(I, S)$ inequalities only upon the base LB. Column With 2 indicates adding reachability inequalities only upon the base LB. Column With 3 indicates adding ATSP-TW inequalities only upon the base LB. Column With 4 indicates adding tournament inequalities only upon the base LB. Column With 5 indicates adding logical inequalities only upon the base LB. Column All indicates adding all five families of cuts upon the base LB.

Table 3. Effects of valid inequalities on CPU seconds at the root node.

\begin{tabular}{lrrrrrrr}
\hline Instance & $\begin{array}{c}\text { With 1 } \\
(\Delta \mathrm{CPU})\end{array}$ & $\begin{array}{c}\text { With 1 } \\
(\Delta \mathrm{CPU})\end{array}$ & $\begin{array}{c}\text { With 2 } \\
(\Delta \mathrm{CPU})\end{array}$ & $\begin{array}{c}\text { With 3 } \\
(\Delta \mathrm{CPU})\end{array}$ & $\begin{array}{c}\text { With 4 } \\
(\Delta \mathrm{CPU})\end{array}$ & $\begin{array}{c}\text { With 5 } \\
(\Delta \mathrm{CPU})\end{array}$ & $\begin{array}{r}\text { All } \\
(\Delta C P U)\end{array}$ \\
\hline C.25-t3 & 0.00 & 0.01 & 0.43 & 0.24 & -0.04 & -0.19 & 0.37 \\
R.25-t3 & 0.01 & 0.01 & 0.02 & 0.15 & 0.00 & -0.21 & 0.53 \\
RC.25-t3 & 0.05 & 0.17 & 0.08 & 0.35 & 0.42 & 0.26 & 0.78 \\
C.30-t3 & 0.02 & 0.17 & 1.70 & 1.69 & 0.03 & -0.23 & 3.59 \\
R.30-t3 & 0.00 & 0.01 & 0.00 & 0.14 & 0.01 & -0.09 & 1.11 \\
RC.30-t3 & 0.08 & 0.03 & -0.29 & -0.06 & 0.79 & 0.55 & 2.34 \\
C.40-t3 & 0.06 & 0.06 & 1.10 & 4.14 & -0.27 & -0.20 & 6.41 \\
R.40-t3 & 1.17 & 1.80 & 0.35 & 0.70 & -0.23 & -0.50 & 2.91 \\
RC.40-t3 & 0.83 & 1.79 & 0.72 & 0.78 & 0.33 & -0.65 & 3.90 \\
C.50-t3 & 0.94 & 2.22 & 6.72 & 8.80 & 0.25 & -0.84 & 8.02 \\
R.50-t3 & 0.59 & 0.59 & -0.68 & -0.51 & 0.38 & -1.84 & 2.56 \\
RC.50-t3 & 0.74 & 0.91 & -0.80 & -0.24 & 4.41 & 2.16 & 8.09 \\
C.100-t3 & -2.51 & -7.66 & 43.35 & 5.52 & 3.53 & -28.91 & 64.60 \\
R.100-t3 & 15.16 & 24.56 & 6.08 & -6.75 & -8.61 & -46.68 & 159.08 \\
RC.100-t3 & 7.98 & 24.55 & 5.91 & -6.99 & 62.85 & 8.24 & 232.47 \\
\hline
\end{tabular}

Notes: The comparing base is the lower bound (LB) obtained from the LP relaxation of the MILP for the PRPTW, including only subtour elimination and incompatible path inequalities. Column With $1^{\prime}$ indicates adding inventory-related inequalities (Archetti et al. 2011) only upon the base LB. Column With 1 indicates adding generalized $(I, S)$ inequalities only upon the base LB. Column With 2 indicates adding reachability inequalities only upon the base LB. Column With 3 indicates adding ATSP-TW inequalities only upon the base LB. Column With 4 indicates adding tournament inequalities only upon the base LB. Column With 5 indicates adding logical inequalities only upon the base LB. Column All indicates adding all five families of cuts upon the base LB.

\subsubsection{The effectiveness of the valid inequalities}

We turn off the proposed heuristic procedure and all the CPLEX cuts and heuristics when we test the effectiveness of valid inequalities on average lower bounds at the root node. The lower bound of the reference state is calculated as the objective value of LP relaxation of the MILP for the PRPTW, including subtour elimination and incompatible path inequalities. 
The inclusion of subtour elimination and incompatible path inequalities ensures that the lower bounds are truly linked with feasible solutions.

The proposed valid inequalities are appended as follows. First, we add generalized $(I, S)$ inequalities only upon the reference state. The results show that generalized $(I, S)$ inequalities turn out to be very effective when customer number is over 50 for all three instance classes. Average lower bounds increase by $11.12 \%$. As a comparison, we also test the effectiveness of related valid inequalities (26) (Archetti et al. 2011). Average lower bounds increase by $7.42 \%$. Thus the proposed generalized $(I, S)$ inequalities outperform previously related valid cuts.

Second, we add strengthened reachability inequalities only upon the reference state. The strengthened reachability inequalities also turn out to be highly effective for all three instance classes, especially when customer number is above 50. Average lower bounds increase as high as $17.36 \%$.

Third, we add ATSP-TW inequalities only upon the reference state. The generalized ATSPTW inequalities turn out to be effective for most of the cases, although average lower bounds increase no more than $0.87 \%$.

Next, we add tournament inequalities only upon the reference state. The generalized tournament inequalities turn out to be only modest effective, because average lower bounds increase no more than $0.2 \%$. The reason might be that the effectiveness of tournament inequalities is offset by the existence of incompatible path inequalities in the reference state.

Then, we add the newly proposed logical inequalities upon the reference state. The new logical inequalities turn out to be effective in all three instance classes. Average lower bounds increase $6.36 \%$ at most.

Finally, we add all the valid inequalities upon the reference state. The computational results show that, for the clustered class, average lower bounds can increase by $19.14 \%$ at most. For the random class, average lower bounds can increase by $29.58 \%$ at most. For the randomly clustered class, average lower bounds can increase by $29.96 \%$ at most.

From Table 3, the computational times are sometimes decreased. The overall increases in computational times are negligible compared with the increase of lower bounds. Thus the computational results show that the valid inequalities we propose are very effective on average.

\subsubsection{The performance of the branch-and-cut algorithm}

We check the performance of the proposed branch-and-cut algorithm on multiple instances. We turn on CPLEX cuts and heuristics by default. The computational results are presented in Tables 4-7. Column Heuristic Time indicates CPU seconds spent by the hybrid heuristic. Column Root Time indicates time spent at the root node. Root time contains heuristic time. Column Branch Time indicates time spent by branching. Column B\&C time indicates the total time spent at the root node and in branching. Column Heur UB indicates heuristic upper bounds obtained by the hybrid heuristic. Column Root LB indicates the lower bounds obtained after solving the root node. Column ObjValue indicates the final upper bounds of the feasible solutions. Column Gap\% indicates the final gaps between the ObjValue and the final lower bounds. Column CPLEX Cuts \# indicates the number of cuts added by CPLEX. Column User Cuts \# indicates the number of cuts added by the proposed 
Table 4. Performance of the proposed branch-and-cut algorithms under formulations I and II.

\begin{tabular}{llcccc}
\hline & \multicolumn{2}{c}{ Formulation I } & & \multicolumn{2}{c}{ Formulation II } \\
\cline { 2 - 3 } Instance & Gap (\%) & CPU (seconds) & & Gap (\%) & CPU (seconds) \\
\hline C.25.t3 & 0 & 28 & 0 & 232 \\
R.25.t3 & 0 & 8 & 0 & 358 \\
RC.25.t3 & 0 & 14 & 0 & 876 \\
C.30.t3 & 0 & 2302 & 0 & 389 \\
R.30.t3 & 1.5 & 3870 & 1.1 & 1600 \\
RC.30.t3 & 3.4 & 14,400 & 0.0 & 1243 \\
C.40.t3 & 0 & 3922 & 0 & 1187 \\
R.40.t3 & 1 & 3837 & 1 & 1438 \\
RC.40.t3 & 3.9 & 14,400 & 0.0 & 1378 \\
C.50.t3 & 0.80 & 3851 & 0.5 & 2257 \\
R.50.t3 & 0 & 1119 & 0.5 & 3600 \\
RC.50.t3 & 1.74 & 14,400 & 1.0 & 1843 \\
C.100.t3 & & Out of memory & 0.64 & 3296 \\
R.100.t3 & & Out of memory & 3.30 & 3600 \\
RC.100.t3 & & Out of memory & 0.81 & \\
\hline
\end{tabular}

Table 5. Performance of the proposed branch-and-cut algorithm with formulation II.

\begin{tabular}{lccrrrrrrrr}
\hline Instance \# & $\begin{array}{c}\text { Heuristic } \\
\text { time }\end{array}$ & $\begin{array}{c}\text { Root } \\
\text { time }\end{array}$ & $\begin{array}{c}\text { Branch } \\
\text { time }\end{array}$ & $\begin{array}{r}\text { B\&C } \\
\text { time }\end{array}$ & $\begin{array}{c}\text { Heur UB } \\
(\%)\end{array}$ & $\begin{array}{c}\text { Root LB } \\
(\%)\end{array}$ & Gap (\%) & $\begin{array}{r}\text { CPLEX } \\
\text { Cuts\# }\end{array}$ & $\begin{array}{r}\text { User } \\
\text { Cuts\# }\end{array}$ & Nodes \# \\
\hline C.25.t3 & 170 & 175 & 57 & 232 & 0.64 & 0.75 & 0 & 146 & 96 & 6973 \\
R.25.t3 & 218 & 223 & 136 & 358 & 1.44 & 1.63 & 0 & 153 & 104 & 16,892 \\
RC.25.t3 & 600 & 608 & 271 & 876 & 1.76 & 1.00 & 0 & 227 & 156 & 21,573 \\
C.30.t3 & 376 & 379 & 10 & 389 & 2.20 & 0.24 & 0 & 165 & 60 & 795 \\
R.30.t3 & 495 & 496 & 1104 & 1600 & 2.68 & 2.67 & 1.09 & 179 & 549 & 102,802 \\
RC.30.t3 & 600 & 616 & 578 & 1243 & 2.51 & 2.10 & 0 & 215 & 161 & 25,179 \\
C.40.t3 & 433 & 440 & 747 & 1187 & 1.67 & 0.54 & 0.13 & 253 & 132 & 19,814 \\
R.40.t3 & 528 & 533 & 905 & 1438 & 1.65 & 1.84 & 1.10 & 218 & 244 & 26,966 \\
RC.40.t3 & 600 & 603 & 355 & 1006 & 8.59 & 1.23 & 0 & 182 & 47 & 2755 \\
C.50.t3 & 477 & 479 & 899 & 1378 & 5.72 & 1.34 & 0.52 & 205 & 113 & 20,147 \\
R.50.t3 & 625 & 628 & 1424 & 2257 & 8.82 & 4.43 & 0.51 & 331 & 304 & 13,663 \\
RC.50.t3 & 600 & 602 & 1961 & 3600 & 6.17 & 2.04 & 1.00 & 404 & 281 & 50,820 \\
C.100.t3 & 676 & 888 & 955 & 1843 & 2.65 & 1.02 & 0.64 & 370 & 125 & 2662 \\
R.100.t3 & 753 & 803 & 2102 & 3296 & 9.89 & 3.33 & 3 & 560 & 316 & 6647 \\
RC.100.t3 & 692 & 838 & 1196 & 3600 & 8.30 & 1.57 & 1 & 440 & 292 & 7639 \\
\hline
\end{tabular}

Table 6. Performance of the proposed branch-and-cut algorithm with formulation II under different inventory holding cost.

\begin{tabular}{|c|c|c|c|c|c|c|c|c|c|c|c|}
\hline Instance \# & $h$ & $\begin{array}{c}\text { Heuristic } \\
\text { time }\end{array}$ & $\begin{array}{l}\text { Root } \\
\text { time }\end{array}$ & $\begin{array}{l}\text { Branch } \\
\text { time }\end{array}$ & $\begin{array}{c}B \& C \\
\text { time }\end{array}$ & $\begin{array}{l}\text { Heur } \\
\text { UB\%\% }\end{array}$ & $\begin{array}{l}\text { Root } \\
\text { LB\% }\end{array}$ & $\begin{array}{l}\text { Gap } \\
(\%)\end{array}$ & $\begin{array}{l}\text { CPLEX } \\
\text { Cuts\# }\end{array}$ & $\begin{array}{l}\text { User } \\
\text { Cuts\# }\end{array}$ & Nodes \# \\
\hline C. $25 . t 3$ & {$[0.1,1]$} & 170.3 & 174.5 & 57 & 232 & 0.64 & 0.75 & 0 & 146 & 96 & 6973 \\
\hline R.25.t3 & {$[0.1,1]$} & 218.0 & 222.5 & 136 & 358 & 1.44 & 1.63 & 0 & 153 & 104 & 16,892 \\
\hline $\mathrm{RC} .25 . \mathrm{t} 3$ & {$[0.1,1]$} & 600.0 & 607.6 & 271 & 876 & 1.76 & 1.00 & 0 & 227 & 156 & 21,573 \\
\hline C.25.t3 & {$[0.01,0.1]$} & 234.0 & 235.0 & 40 & 275 & 2.06 & 1.49 & 0 & 105 & 55 & 3573 \\
\hline R.25.t3 & {$[0.01,0.1]$} & 224.3 & 229.3 & 853 & 1082 & 3.47 & 2.63 & 0.37 & 159 & 584 & 51,071 \\
\hline RC.25.t3 & {$[0.01,0.1]$} & 149.9 & 87.8 & 1238 & 1391 & 3.49 & 2.18 & 0 & 193 & 513 & 83,746 \\
\hline
\end{tabular}

branch-and-cut algorithm. Column Nodes \# indicates the number of nodes processed by the algorithm.

First, we compare the performance of the branch-and-cut algorithm with formulations I and II in Table 4. For instances with less than 50 customers, the branch-and-cut algorithm with formulation I is faster than the algorithm with formulation II for most cases. However, 
Table 7. Performance of the proposed branch-and-cut algorithm with formulation II under different planning horizons.

\begin{tabular}{lccccccrr}
\hline Instance \# & Heuristic time & B\&C time & Heur UB\% & Root LB\% & Gap (\%) & CPLEX Cuts\# & User Cuts\# & Nodes \# \\
\hline C.25.t3 & 126 & 126 & 1.00 & 0.47 & 0 & 60 & 17 & 20 \\
R.25.t3 & 72 & 75 & 3.95 & 0.68 & 0 & 90 & 42 & 153 \\
RC.25.t3 & 685 & 1894 & 5.66 & 2.55 & 0.32 & 305 & 175 & 32,711 \\
C.50.t3 & 300 & 302 & 17.17 & 1.10 & 0 & 112 & 13 & 5 \\
R.50.t3 & 651 & 2115 & 10.64 & 8.06 & 0.24 & 311 & 406 & 17,164 \\
RC.50.t3 & 600 & 3600 & 7.36 & 2.24 & 0.81 & 507 & 228 & 74,194 \\
C.25.t6 & 1341 & 2311 & 1.51 & 0.77 & 0.23 & 193 & 51 & 40,422 \\
R.25.t6 & 620 & 2160 & 1.72 & 1.26 & 0.68 & 475 & 211 & 31,255 \\
RC.25.t6 & 1207 & 3600 & 1.05 & 1.50 & 1.01 & 440 & 268 & 62,981 \\
C.50.t6 & 702 & 3600 & 12.98 & 1.32 & 1.09 & 475 & 80 & 17,078 \\
R.50.t6 & 753 & 3600 & 4.32 & 5.67 & 5.44 & 838 & 353 & 10,098 \\
RC.50.t6 & 844 & 3600 & 9.13 & 4.61 & 9.52 & 621 & 326 & 13,893 \\
\hline
\end{tabular}

for some instances, especially those which cannot be solved by both algorithms, the proposed branch-and-cut algorithm can obtain a better lower bound under formulation II. For instances over 50 customers, the branch-and-cut algorithm with formulation I runs out of memory. Whereas the proposed branch-and-cut algorithm with formulation II can still solve some of the instances to optimality.

The details on the performance of the proposed branch-and-cut algorithm with formulation II are given in Tables 5-7. For the basic configuration, we solve all instances on 25 customers, 3 periods in 1 hour. The instances with 30 customers are solved for 11 out of 12 cases. The unsolved instances have average gaps $4.3 \%$. The instances with 40 customers are solved for 10 out of 12 cases. The unsolved instances have average gaps $4.4 \%$. The instances with 50 customers are solved for 5 out of 12 instances. The unsolved instances have average gaps $2.1 \%$. The instances with 100 customers are solved for 4 out of 12 instances. The unsolved instances have average gaps $9.87 \%$.

We also check the performance of the branch-and-cut algorithm under different inventory holding costs. As shown in Table 6, the branch-and-cut algorithms perform almost equally under these two situations, although one of the instances with smaller inventory cost is not solved within 1 hour. The average gap is $1.48 \%$. The hybrid heuristic has met with difficulty in this instance to find a good enough solution.

Finally, we check the performance of the branch-and-cut algorithm under different planning horizons. As the planning horizon increases to $|T|=6$, the branch-and-cut algorithm solves two instances in 1 hour. The unsolved instances have average gaps at most $2 \%$ when customer size is less than 50 .

\section{Case study}

This section presents an implementation of the proposed model on the production and distribution operations of a food company operating in the Nanjing city, China. We first describe the case study, then present our results.

\subsection{Description and data}

In the Nanjing city, China, most consumer products are distributed through chain stores of power retailers. Suguo company is among the biggest power retailers. Suguo covers the 


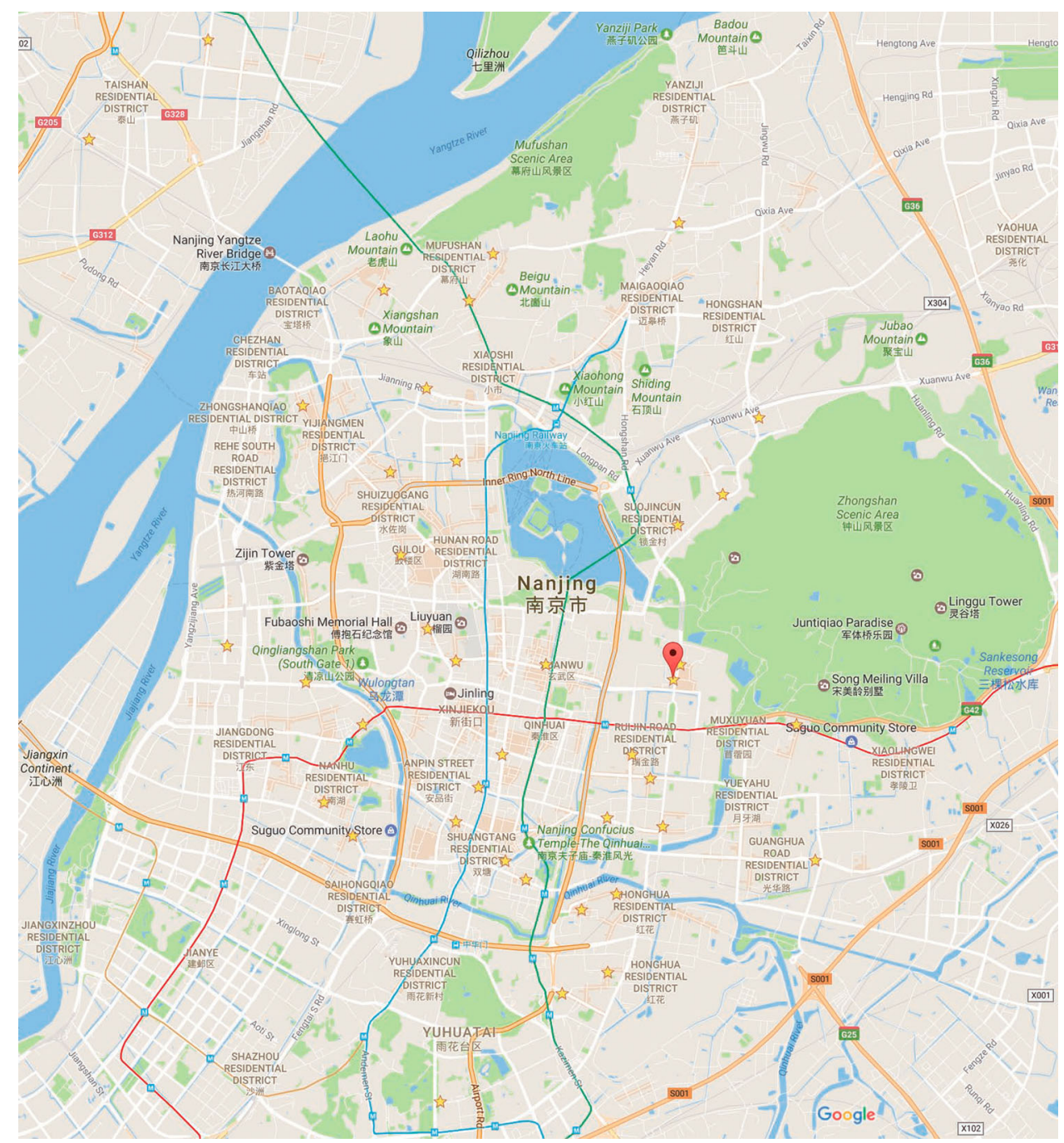

Figure 1. The representation of the retailer networks.

city with hundreds of large and small stores. Suguo community stores are large and thus the target store of the food company. The food company, situated on the north side of the Yangtze river, provides a certain kind of fresh meat product to Suguo community stores which mainly located on the south side of the Yangtze river. The retailer network is illustrated in Figure 1, in which the retailers are represented by yellow stars. The shortest paths between these sites are calculated using Google Maps. Detailed information can be found in the online supplement.

In a typical operations scenario, the food company has only $200 \mathrm{~kg}$ of fresh meat products in stock at the beginning of planning period. The 40 Suguo community stores have only limited products in stock at the beginning of planning horizon. According to the purchasing statistics, the Suguo community stores have demand estimates for the product for the following week. The vehicle used for deliveries can carry 8 tons of meat products and traverse 
the city at an average speed of 40 kilometers per hour. The cost to distribute these products is $30 \mathrm{RMB}$ dollars per kilometer. Other parameters are included in the online supplement.

Since the city of Nanjing has a population of nearly 10 million, traffic condition is getting worse. Consequently, the retailer stores can only be served within relative narrow time windows. The food company will decide how many items to produce, and the routing plans which respect the time windows.

\subsection{Results}

Before we apply the proposed model and solution method, the company made use of a heuristic solution, which results in a cost of 400,655 RMB dollars for the planning week. Using our model and algorithm, we can provide an optimized solution with a cost of 354,065 RMB dollars within seconds, resulting a $11.6 \%$ of decrease.

\section{Conclusion}

We have introduced, modeled, and analyzed the production routing problem with time windows (PRPTW), a generalization of the multi-vehicle production and inventory routing problem. We have developed a new model for the PRPTW which incorporates time windows and lot-sizing ingredients of the PRPTW. We also have introduced several families of valid inequalities: logical, lot-sizing, and vehicle routing types (specifically asymmetric traveling salesman problem with time windows and vehicle routing problem with time windows). These inequalities greatly increase the lower bounds at the root of the branchand-cut tree and reduce the CPU times for finding optimal PRPTW solutions. Numerical results from the case study also show that the algorithm has the potential to reduce the total costs.

Several generalizations are possible for the PRPTW. One worth mentioning here is the possibility of incorporating start-up times when there are multiple types of products. Another would be to consider the problem in more complex supply chain environments like multi-level production and routing problems with time windows. The branch-andcut algorithm can be further improved by incorporating column generation techniques to reduce the CPU times when dealing with a larger number of time periods. A comparative study of the strong branching rule and different bottleneck tolerance based rules (Goldengorin et al. 2006) might be beneficial to reduce the CPU times for our branch-and-cut algorithm.

\section{Acknowledgments}

We would like to thank the anonymous reviewers for their constructive and insightful suggestions. The programming help from Dr. Jack Gao is also acknowledged.

\section{Disclosure statement}

No potential conflict of interest was reported by the authors. 


\section{Funding}

Y. Qiu's work is supported by National Natural Science Foundation of China (NSFC) under Grant No. 71571092. Y. Qiu also acknowledges the support from Jiangsu Overseas Research \& Training Program for University Prominent Young \& Middle-aged Teachers and Presidents during her stay at UF. Y. Qiu's work is also supported in part by General Research Project for Humanities and Social Sciences from Chinese Ministry of Education under Grant No. 11YJCZH137 and the Priority Academic Program Development of Jiangsu Higher Education Institutions (PAPD).

\section{ORCID}

Yuzhuo Qiu (D) http://orcid.org/0000-0002-9772-1546

\section{References}

Absi, N., C. Archetti, S. Dauzere-Peres, and D. Feillet. 2015. "A Two-Phase Iterative Heuristic Approach for the Production Routing Problem." Transportation Science 49 (4): 784-795.

Achterberg, T., T. Koch, and A. Martin. 2005. "Branching Rules Revisited." Operations Research Letters 33 (1): 42-54.

Adulyasak, Y., J.-F. Cordeau, and R. Jans. 2014a. "Formulations and Branch-and-Cut Algorithms for Multivehicle Production and Inventory Routing Problems." INFORMS Journal on Computing 26 (1): 103-120.

Adulyasak, Y., J.-F. Cordeau, and R. Jans. 2014b. "Optimization-Based Adaptive Large Neighborhood Search for the Production Routing Problem." Transportation Science 48 (1): 20-45.

Adulyasak, Y., J.-F. Cordeau, and R. Jans. 2015a. "Benders Decomposition for Production Routing Under Demand Uncertainty." Operations Research 63 (4): 851-867.

Adulyasak, Y., J.-F. Cordeau, and R. Jans. 2015b. "The Production Routing Problem: A Review of Formulations and Solution Algorithms." Computers \& Operations Research 55, 141-152.

Archetti, C., L. Bertazzi, G. Laporte, and M. G. Speranza. 2007. "A Branch-and-Cut Algorithm for a Vendor-Managed Inventory-Routing Problem." Transportation Science 41 (3): 382-391.

Archetti, C., L. Bertazzi, G. Paletta, and M. G. Speranza. 2011. "Analysis of the Maximum Level Policy in a Production-Distribution System." Computers \& Operations Research 38 (12): 1731-1746.

Armentano, V. A., A. L. Shiguemoto, and A. Lokketangen. 2011. "Tabu Search with Path Relinking for an Integrated Production-Distribution Problem." Computers \& Operations Research 38 (8): 1199-1209.

Bard, J. F., G. Kontoravdis, and G. Yu. 2002. "A Branch-and-Cut Procedure for the Vehicle Routing Problem with Time Windows." Transportation Science 36 (2): 250-269.

Bard, J. F., and N. Nananukul. 2009a. "Heuristics for a Multi-Period Inventory Routing Problem with Production Decisions." Computers \& Industrial Engineering 57 (3): 713-723.

Bard, J. F., and N. Nananukul. 2009b. "The Integrated Production-Inventory-Distribution-Routing Problem." Journal of Scheduling 12 (3): 257-280.

Bard, J. F., and N. Nananukul. 2010. "A Branch-and-Price Algorithm for an Integrated Production and Inventory Routing Problem." Computers \& Operations Research 37 (12): 2202-2217.

Boudia, M., M. A. O. Louly, and C. Prins. 2007. "A Reactive Grasp and Path Relinking for a Combined Production-Distribution Problem." Computers \& Operations Research 34 (11): 3402-3419.

Boudia, M., and C. Prins. 2009. "A Memetic Algorithm with Dynamic Population Management for an Integrated Production-Distribution Problem." European Journal of Operational Research 195 (3): 703-715.

Chandra, P., and M. L. Fisher. 1994. "Coordination of Production and Distribution Planning." European Journal of Operational Research 72 (3): 503-517.

Chow, J. Y., and A. E. Nurumbetova. 2015. "A Multi-Day Activity-Based Inventory Routing Model with Space-Time-Needs Constraints." Transportmetrica A: Transport Science 11 (3): 243-269.

Díaz-Madroñero, M., D. Peidro, and J. Mula. 2015. "A Review of Tactical Optimization Models for Integrated Production and Transport Routing Planning Decisions." Computers \& Industrial Engineering $88,518-535$. 
Fischetti, M., and P. Toth. 1997. "A Polyhedral Approach to the Asymmetric Traveling Salesman Problem." Management Science 43 (11): 1520-1536.

Fumero, F., and C. Vercellis. 1999. "Synchronized Development of Production, Inventory, and Distribution Schedules." Transportation Science 33 (3): 330-340.

Goldengorin, B., G. Jager, and P. Molitor. 2006. "Tolerance Based Contract-or-Patch Heuristic for the Asymmetric TSP." Lecture Notes in Computer Science 4235: 86-97.

Kallehauge, B., N. Boland, and O. B. G. Madsen. 2007. "Path Inequalities for the Vehicle Routing Problem with Time Windows." Networks 49 (4): 273-293.

Kumar, R. S., K. Kondapaneni, V. Dixit, A. Goswami, L. S. Thakur, and M. K. Tiwari. 2016. “Multi-Objective Modeling of Production and Pollution Routing Problem with Time Window: A Self-Learning Particle Swarm Optimization Approach." Computers \& Industrial Engineering 99, 29-40.

Lei, L., S. Liu, A. Ruszczynski, and S. Park. 2006. "On the Integrated Production, Inventory, and Distribution Routing Problem." IIE Transactions 38 (11): 955-970.

Lysgaard, J. 2006. "Reachability Cuts for the Vehicle Routing Problem with Time Windows." European Journal of Operational Research 175 (1): 210-223.

Lysgaard, J., A. N. Letchford, and R. W. Eglese. 2004. "A New Branch-and-Cut Algorithm for the Capacitated Vehicle Routing Problem." Mathematical Programming 100 (2): 423-445.

Miller, C. E., A. W. Tucker, and R. A. Zemlin. 1960. "Integer Programming Formulation of Traveling Salesman Problems." Journal of the ACM (JACM) 7 (4): 326-329.

Naddef, D., and G. Rinaldi. 2001. "Branch-and-Cut Algorithms for the Capacitated VRP." In The Vehicle Routing Problem, edited by P. Toth, D. Vigo, 53-84. Philadelphia, PA: Society for Industrial and Applied Mathematics.

Pochet, Y., and L. A. Wolsey. 2006. Production Planning by Mixed Integer Programming. New York: Springer-Verlag.

Qiu, Y., J. Qiao, and P. M. Pardalos. 2017. "A Branch-and-Price Algorithm for Production Routing Problems with Carbon Cap-and-Trade." Omega 68, 49-61.

Savelsbergh, M. W. P. 1985. "Local Search in Routing Problems with time Windows." Annals of Operations Research 4 (1): 285-305.

Solomon, M. M. 1987. "Algorithms for the Vehicle Routing and Scheduling Problems with Time Window Constraints." Operations Research 35 (2): 254-265.

Toth, P., and V. Daniele. 2014. Vehicle Routing: Problems, Methods, and Applications. Philadelphia, PA: SIAM.

You, S. I., J. Y. J. Chow, and S. G. Ritchie. 2016. "Inverse Vehicle Routing for Activity-Based Urban Freight Forecast Modeling and City Logistics." Transportmetrica A: Transport Science 12 (7): 650-673. 